\title{
Chemical resistance of core-shell particles (PS/PMMA) polymerized by seeded suspension
}

\author{
Luiz Fernando Belchior Ribeiro', Odinei Hess Gonçalves ${ }^{2}$, Cintia Marangoni ${ }^{3}$, Günter Motz ${ }^{4}$ and \\ Ricardo Antonio Francisco Machado ${ }^{1 *}$
}

\author{
${ }^{1}$ Materials Engineering Department, Universidade Federal de Santa Catarina - UFSC, \\ Florianópolis, SC, Brazil \\ ${ }^{2}$ Post-graduation Program in Food Technology, Universidade Técnológica Federal do Paraná - UTFPR, \\ Campo Mourão, PR, Brazil \\ ${ }^{3}$ Universidade Federal de Santa Catarina - UFSC, Blumenau, SC, Brazil \\ ${ }^{4}$ Lehrstuhl Keramische Werkstoffe, Universität Bayreuth, Bayreuth, Germany \\ *ricardo.machado@ufsc.br
}

\begin{abstract}
Core-shell particles were produced on seeded suspension polymerization by using polystyrene (PS) as polymer core, or seed, and methyl methacrylate (MMA) as the shell forming monomer. Two synthesis routes were evaluated by varying the PS seed conversion before MMA addition. The main purpose of this work was to investigate the influence of synthesis routes on the morphology and chemical resistance of the resulting particles. ${ }^{1} \mathrm{H}$ NMR spectroscopy showed that the use of PS seeds with lower conversion led to the formation of higher amount of poly(styrene-co-MMA). The copolymer acted as a compatibilizer, decreasing the interfacial energy between both homopolymers. As a consequence, a larger amount of reduced PMMA cluster were formed, as was revealed by TEM measurements. Samples in this system showed enhanced resistance to cyclohexane attack compared with pure PS, with a PS extraction of only $37 \%$ after 54 hours test.
\end{abstract}

Keywords: seeded suspension polymerization, poly(methyl methacrylate), polystyrene, suspension polymerization.

\section{Introduction}

Styrene suspension polymerization has been extensively studied over the past decades ${ }^{[1-5]}$. It consists in polymerizing relatively water-insoluble monomer droplets in a water media formed by vigorous stirring in the presence of a steric stabilizer. The synthesis leads to the formation of spherical polymer particles dispersed in an aqueous system.

The main use of polystyrene (PS) produced by suspension is the production of expandable polystyrene (EPS). EPS foams possess excellent properties, which combine low density with high mechanical resistance. Therefore, EPS foams are used in a wide range of applications, especially in the construction and packaging industries. The range of applications of these foams would be broader by improving some of the EPS properties, in particular their chemical resistance.

Low chemical resistance of PS is a limiting factor for many applications, such as the transport of heavy equipment containing lubricant greases. In this case, the EPS foam needs to be covered with a chemically resistant polymer film, which introduces a subsequent manufacturing step, increases the cost of production and makes the use of it near prohibitive.

The use of poly(methyl methacrylate) (PMMA) foam as a substitute to EPS is reported elsewhere ${ }^{[6]}$. PMMA chemical resistance is much higher than $\operatorname{PS}^{[7]}$ and despite the promising results, the application is limited by the higher cost of monomers.
An interesting way to overcome this problem is the synthesis of core-shell particles using lower amounts of PMMA to cover PS particles. Gonçalves et al. ${ }^{[8]}$ investigated the morphology of core-shell particles composed of PS core and PMMA shell. The synthesis consisted in feeding methyl methacrylate (MMA) monomers with the initiator at low temperature $\left(50^{\circ} \mathrm{C}\right)$ to swell previously obtained PS seeds. In a subsequent step, the temperature reaction is increased up to $70^{\circ} \mathrm{C}$ in order to polymerize the MMA forming shell. The obtained particles presented a complex morphology with a PS core and a shell composed of PMMA domains distributed in the PS matrix. Further works ${ }^{[9]}$ evaluated the expansion of the aforementioned synthesized core-shell particles. The results revealed that the expansion needs higher temperatures than the conventional EPS processing. Moreover, the presence of PMMA at the surface seems to hinder particle expansion, and a non-uniform foamed particle was obtained. This result is in agreement with a more recent study published by Heydarpoor et al. ${ }^{[10]}$. The authors investigated similar systems of PS/PMMA core-shell expandable particles, by using pentane as the expansion agent. They concluded that pentane was more concentrated in the PS core than in the PMMA domains, which was responsible for a non-uniform expansion behavior.

In this work a similar system used by Gonçalves et al. ${ }^{[8]}$ was employed but the PS seed was polymerized in situ. The use of in situ polymerized PS seeds allows to control the polymer conversion before MMA addition, which could be used to change particle morphology by improving the 
MMA diffusion inside the seeds. Two different synthesis route were evaluated and the resulting particles were investigated in respect to their morphology and chemical resistance.

\section{Materials and Methods}

\subsection{Materials}

Technical grade styrene (Innova S.A.) and methyl methacrylate (Rohm\&Hass S.A.) were used as monomers. Benzoyl peroxide (BPO, Sigma-Aldrich) was used as an initiator. Distilled water was used as continuous phase and poly(vinyl pyrrolidone) (PVP K90, Sigma-Aldrich) as stabilizer. Ascorbic acid (Sigma-Aldrich) was used to avoid inhibition caused by oxygen, and sodium chloride PA (Vetec) was used to decrease methyl methacrylate solubility in water. Chloroform-d (Sigma-Aldrich) was used to solubilize samples for ${ }^{1} \mathrm{H}$ NMR investigations and cyclohexane (Vetec) was used for the chemical resistance tests. All chemicals were used as received without further purifications.

\subsection{Core-shell synthesis}

The synthesis of the core-shell particles was carried out in a $5 \mathrm{~L}$ jacketed reactor fitted with stirring rate and temperature control. The synthesis consisted in a two-step reaction and the formulation employed is shown in detail at Table 1. The first step consisted in synthesizing the PS seed using conventional suspension polymerization. Water, styrene and BPO were added in the reactor at room temperature and the system was heated up to $90^{\circ} \mathrm{C}$ and stirred at this temperature until the determined reaction time. PVP was added only $1 \mathrm{~h}$ after the reaction media reached $90^{\circ} \mathrm{C}$. In the second step, ascorbic acid and sodium chloride were added in the reactor and the system was cooled $\left(50^{\circ} \mathrm{C}\right)$. The additional amount of BPO was dissolved in the MMA monomer and feed in the reactor at a constant rate $(5 \mathrm{~g} / \mathrm{min})$. After the monomer/initiator feed completion, the system was heated up to $70^{\circ} \mathrm{C}$ and allowed to react for $4 \mathrm{~h}$. The period of time in which the system was kept at low temperature before reaching $70^{\circ} \mathrm{C}$ was arbitrarily defined as swelling time. During this period, no significant amount of PMMA is expected to be formed.

Two synthesis routes were evaluated by varying the PS seed conversion before MMA addition. The PS seed conversion was determined by reaction time, 7 hours for synthesis route 1 and 5 hours for synthesis route 2 . The choice

Table 1. Formulation used for core-shell particles synthesis.

\begin{tabular}{lc}
\hline \multicolumn{1}{c}{ Reactant } & Weight (g) \\
\hline$I^{a}$ Step $-P S$ seed synthesis & \\
Water & 3624.0 \\
Styrene & 969.0 \\
PVP & 130.0 \\
BPO & 5.3 \\
$2^{a}$ Step- Core-shell synthesis & \\
MMA & 302.0 \\
Ascorbic acid & 5.4 \\
Sodium cloride & 181.2 \\
BPO & 1.7 \\
\hline
\end{tabular}

for reaction times was based on a mathematical model of styrene homopolymerization to obtain PS conversions near to 100 and $90 \%{ }^{[11]}$. The conversion was analyzed by gas chromatography and the values founded were $98 \%$ for synthesis route 1 and $92 \%$ for synthesis route 2 . The temperature profiles used in these reactions are represented in Figures 1a and 1b.

\subsection{Characterizations}

Styrene conversion was investigated by gas chromatography (GC) analysis. A sample of the polystyrene seed was taken from the reaction medium before the addition of MMA and solubilized in chloroform. An aliquot of $20 \mu \mathrm{l}$ was taken from the solution, added in a sealed vial and analyzed with a Shimadzu GC-2010AF (Shimadzu Corporation, Japan) equipped with a headspace auto sampler (Shimadzu AOC-5000), a flame ionization detector at $220^{\circ} \mathrm{C}$ and a Restek $30 \mathrm{~m}$ RTX-5 column. A calibration curve was previously performed to determine the amount of unreacted styrene.

The composition and structural characterization of core-shell particles was investigated by ${ }^{1} \mathrm{H}$ NMR spectroscopy. The samples were solubilized in chloroform-d and the NMR-spectra of the polymer solutions were collected on a Bruker AC200 (200 MHz) spectrometer. The molar percentage of PMMA was calculated using Equation 1.

$$
\% P M M A=\left(\frac{A / 3}{A / 3+B / 5}\right) * 100
$$

where $\mathrm{A}$ is the integrated area related to $\mathrm{CH}_{3}-\mathrm{O}$ peaks in PMMA and $\mathrm{B}$ is the integrated area related to different aromatic protons in PS.

Transmission electron microscopy (JEM-1011 TEM at $100 \mathrm{kV}$ ) was used to investigate the morphology of the particles. The particles were sliced using an ultramicrotome and deposited on a copper grid. The samples were stained by ruthenium tetroxide $\left(\mathrm{RuO}_{4}\right)$ vapor for $1 \mathrm{~h}$ to reveal the phases.

Chemical resistance was tested by particle dissolution in cyclohexane, a selective solvent of PS. For sampling, the agitation was ceased and a liquid phase sample was collected. The collected samples were dried in an oven and the amount of PS extracted was determined gravimetrically.

For all characterization technique employed particles with diameter size in the range of $1.0-1.18 \mathrm{~mm}$ were selected.

\section{Results and Discussions}

\subsection{NMR measurements}

The ${ }^{1} \mathrm{H}$ NMR spectrum (Figure 2) shows the specific signals for PS and PMMA homopolymers for both systems. The resonances at $6.2-7.5 \mathrm{ppm}$ are due to the different aromatic protons in PS while the resonances at 3.2-3.9 ppm are characteristic to the $\mathrm{O}_{-} \mathrm{CH}_{3}$ group of PMMA. In addition to typical peaks from PS and PMMA homopolymers, a new peak appears at $2.9 \mathrm{ppm}$. This signal is commonly assigned to $\mathrm{O}-\mathrm{CH}_{3}$ sites bonded to PS sequences ${ }^{[10,12]}$, which proves that a copolymerization reaction is taking place. By using the integrated area of the peak centered at 2.9, the percent of copolymer formed for synthesis route 1 and 2 were determined to be 1 and $6 \mathrm{~mol} \%$ respectively. The higher 


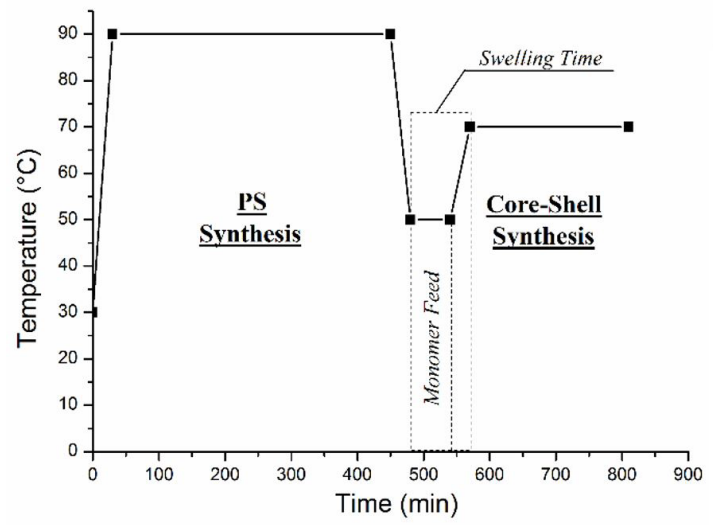

(a)

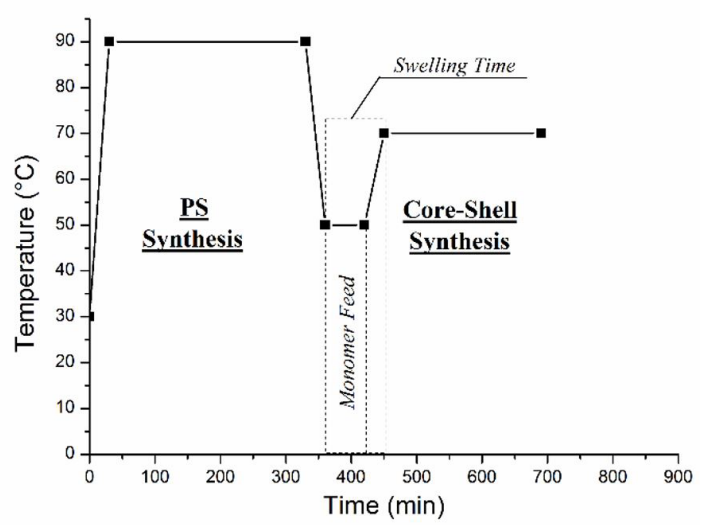

(b)

Figure 1. Temperature profile used in the experiment of (a) synthesis route 1 and (b) synthesis route 2.

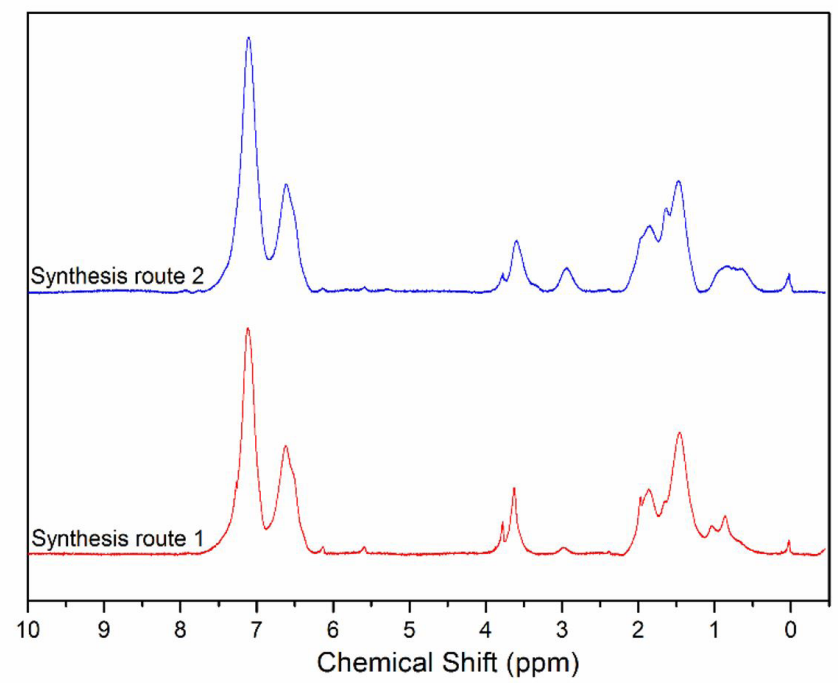

Figure 2. ${ }^{1} \mathrm{H}$ NMR spectrum of the synthesized core-shell particles.

amount of poly(styrene-co-MMA) formed in synthesis route 2 particles is due to the lower PS seed conversion in this system, which contains a higher amount of residual styrene to react with MMA.

The PMMA molar percentages in core-shell particles were calculated as described in the experimental procedure and the results are shown in Table 2. Incorporation efficiency was defined as the ratio of the MMA fed to the reactor and the MMA effectively incorporated into the particles.

There was no significant difference in PMMA incorporation. Both systems presented high incorporation efficiency. For the systems under study, swelling time seems to rule the MMA incorporation in the PS seeds. However, as will be further discussed, morphology was drastically influenced by the conversion of the PS seed.

\subsection{TEM analysis}

TEM micrographs of the core-shell particles are presented in Figure 3a and 3b. On these images, PMMA appears as light gray and PS as dark gray. The cross section
Table 2. Concentration of PMMA and incorporation efficiency of core-shell particles.

\begin{tabular}{ccc}
\hline Synthesis Route & PMMA (mol \%) & $\begin{array}{c}\text { Incorporation } \\
\text { efficiency (\%) }\end{array}$ \\
\hline 1 & 18 & 74 \\
2 & 20 & 82 \\
\hline
\end{tabular}

representation shows the approximated region where the images were obtained.

The TEM measurements reveal that core-shell morphology consisted of PMMA clusters dispersed in the PS matrix and the size and concentration of these clusters decrease along the radius. Previous studies showed that this kind of morphology was expected ${ }^{[8,10]}$. This occurred because the polymerization rate is much faster than the diffusion of PMMA/MMA in the swollen PS particles.

When comparing the TEM images of the synthesis route 1 and 2 particles, it was noteworthy the influence of PS seed conversion in the distribution and size of the PMMA clusters. In the system using a lower PS seed conversion it 

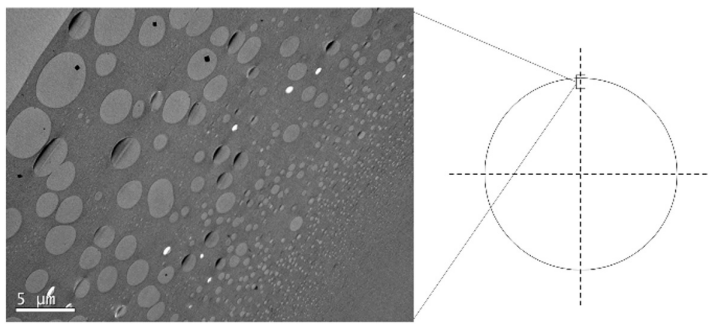

(a)
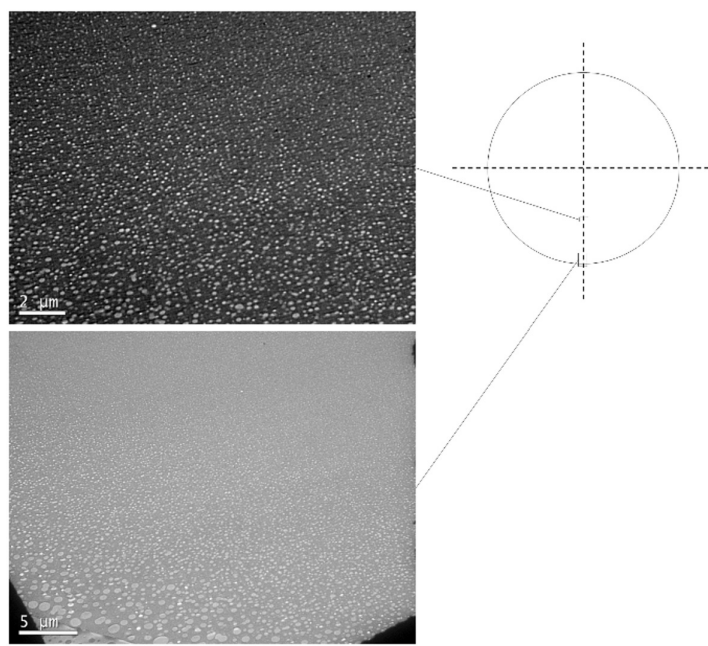

(b)

Figure 3. TEM images and the approximated representation of the cross section of core-shell particles from (a) synthesis route 1 and (b) synthesis route 2 .

was possible to identify the presence of PMMA domains closer to the center of the particle (Figure 4b). By contrast, for the particles from the system employing higher PS seed conversion, the PMMA clusters are more concentrated at the surface. These differences can be explained in part by the higher diffusion rate of MMA in particles from synthesis route 2 . In this system, the higher amount of residual styrene present in PS particles act as a solvent. As a result, intermolecular forces between the PS chains are decreased, which improve MMA diffusion and allows the nucleation of PMMA clusters closer to the center of the particle. In addition, the formation of a poly(styrene-co-MMA), confirmed by ${ }^{1} \mathrm{H}$ NMR measurements, reduced the PS/PMMA interfacial energy. The presence of a higher amount of copolymers in particles from synthesis route 2 act as a compatibilizer, and it was responsible for the formation of a higher amount of reduced size clusters.

\subsection{Chemical resistance tests}

The chemical resistance results are shown in Figure 4. The percentage of polystyrene extracted by the solvent (cyclohexane) was determined gravimetrically in relation with the total of PS present in the particles. All the measurements were made in triplicate.

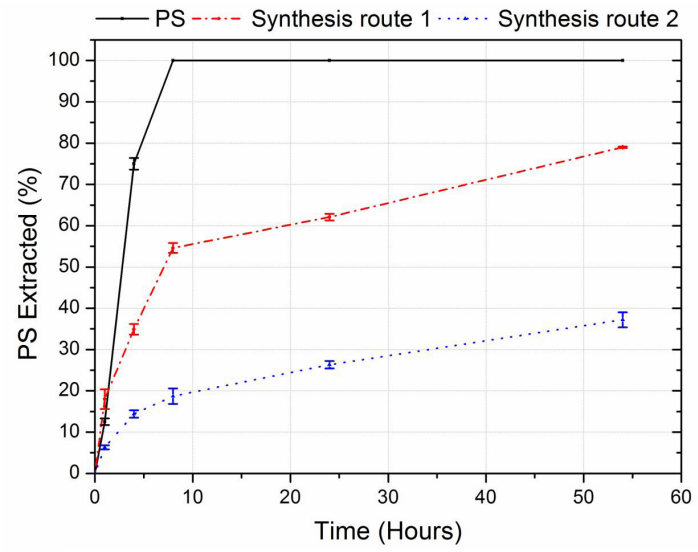

Figure 4. Polystyrene extraction by dissolution in cyclohexane.

The core-shell morphology for both reaction systems improved cyclohexane resistance in comparison to pure PS. After $8 \mathrm{~h}$ tests, the pure PS particles were completely solubilized. In contrast, for core-shell particles derived from synthesis route 1 and 2, only 55 and $19 \%$ of PS was extracted respectively. As cyclohexane is a non-solvent to PMMA, its presence at the particle surface hinder solvent diffusion, thus retarding PS core extraction. Even after long period test, the PS were not completely extracted in core-shell particles, which reflect the improvement in chemical resistance as the benefit of the presence of PMMA clusters at the particle surface. The remarkable result for particles from synthesis route 2 is clearly justified by their morphology, which contains more PMMA clusters densely grouped at the surface.

\section{Conclusions}

In this work, core-shell particles were produced through seeded suspension polymerization by using polystyrene (PS) as a seed, and methyl methacrylate (MMA) as the shell forming monomer. The core-shell particles presented a complex morphology with a PS core and a shell composed by PMMA clusters distributed in the PS matrix. The size and concentration of these clusters along the particle radius were highly influenced by the styrene conversion. The use of PS seed with a lower conversion promoted MMA diffusion inside the PS seeds, and PMMA clusters were observed near the center of the particle. Moreover, the formation of poly(styrene-co-MMA) decreased the PS/PMMA interfacial energy, which led to formation of smaller PMMA clusters.

Finally, the synthesized core-shell particles presented enhanced chemical resistance compared to PS. The presence of PMMA domains grouped at the particles surface prevents the completely PS extraction, even after long period tests.

\section{Acknowledgements}

The authors would lilke to thank CAPES-PROBRAL and CAPES-PVE (Coordination for the Improvement of Higher Education Personnel) and CNPq (National Council for Scientificand Technological Development) for their financial support. We are also thankful LCME 
- UFSC (Electron Microscope Laboratory) for the TEM measurements.

\section{References}

1. Vivaldo-Lima, E., Wood, P. E., Hamielec, A. E., \& Penlidis, A. (1997). An updated review on suspension polymerization. Industrial \& Engineering Chemistry Research, 36(4), 939-965. http://dx.doi.org/10.1021/ie960361g.

2. Dowding, P. J., \& Vincent, B. (2000). Suspension polymerisation to form polymer beads. Colloids and Surfaces. A, Physicochemical and Engineering Aspects, 161(2), 259-269. http://dx.doi. org/10.1016/S0927-7757(99)00375-1.

3. Scheirs, J., \& Priddy, D. (2003). Modern styrenic polymers: polystyrenes and styrenic copolymers. Chichester: John Wiley \& Sons, Ltd.

4. Brooks, B. W. (2005). Free-radical polymerization: suspension. In T. H. Meyer \& J. Keurentjes (Eds.), Handbook of Polymer Reaction Engineering (pp. 213-247). Weinheim: Wiley-VCH Verlag $\mathrm{GmbH}$.

5. Kotoulas, C., \& Kiparissides, C. (2008). Suspension polymerization. In J. Asua (Ed.), Polymer Reaction Engineering (pp. 209-232). Oxford: Blackwell Publishing Ltd.

6. Gonçalves, O. H., Staudt, T., Araújo, P. H. H., \& Machado, R. A. F. (2009). Foaming of poly(methyl methacrylate) particles. Materials Science and Engineering C, 29(2), 479-484. http:// dx.doi.org/10.1016/j.msec.2008.08.034
7. Brandrup, J., Immergut, E. H., \& Grulke, E. A. (1999). Polymer handbook. New York: John Wiley \& Sons, Inc.

8. Gonçalves, O. H., Asua, J. M., Araújo, P. H. H., \& Machado, R. A. F. (2008). Synthesis of PS/PMMA core-shell structured particles by seeded suspension polymerization. Macromolecules, 41(19), 6960-6964. http://dx.doi.org/10.1021/ma800693m.

9. Gonçalves, O. H., Leimann, F. V., Araújo, P. H. H., \& Machado, R. A. F. (2013). Expansion of core-shell PS/PMMA particles. Journal of Applied Polymer Science, 130(6), 4521-4527. http:// dx.doi.org/10.1002/app.39731.

10. Heydarpoor, S., Abbasi, F., Jalili, K., \& Najafpour, M. (2015). Synthesis of core-shell PS/PMMA expandable particles via seeded suspension polymerization. Journal of Polymer Research, 22(8), 151. http://dx.doi.org/10.1007/s10965-015-0789-0.

11. Machado, R. A. F., \& Bolzan, A. (1998). Control of batch suspension polymerization reactor. Chemical Engineering Journal, 70(1), 1-8. http://dx.doi.org/10.1016/S1385-8947(98)00006-0.

12. Songkhla, P. N., \& Wootthikanokkhan, J. (2002). Effect of the copolymer composition on the $\mathrm{K}$ and a constants of the Mark-Houwink equation: Styrene-methyl methacrylate random copolymers. Journal of Polymer Science. Part B, Polymer Physics, 40(6), 562-571. http://dx.doi.org/10.1002/polb.10119.

Received: Oct. 25, 2016

Revised: Feb. 13, 2017

Accepted: Mar. 02, 2017 